\title{
Uso de LinkedIn como herramienta docente en el Máster de Producción Animal
}

\author{
María Cambra-López, Fernando Estellés Barber, Juan José Pascual Amorós, \\ Cristofol Peris Ribera, Salvador Calvet Sanz \\ Departamento de Ciencia Animal, Universitat Politècnica de València. e-mail \\ macamlo@upvnet.upv.es
}

\begin{abstract}
LinkedIn professional social network was used as a common platform for exposure, discussion and analysis of information. Its use can facilitate collaborative learning of students, stimulate their motivation and enhance their professional career project. The study was conducted in three subjects of the Master in Animal Production at the Universitat Politècnica de València during 2014-2015 academic year. The specific objectives were: i) to create a virtual learning community, ii) to facilitate student's active participation, iii) to enhance teacher-student and student-student interactions, iv) to promote critical thinking, and v) to encourage the use of this professional network that favors student's professional career plan. Four actions based on the development and maintenance of a LinkedIn group and the ellaboration of a heading of critical thinking were established. The achievement of the objectives was assessed by the instrinsic network's activity, as well as by quantitative and qualitative evaluations and a survey of self perception. All students created and used their profile regularly. Discussion topics were proposed, assessing student's participation during the study. The difficulty of evaluating the development of critical thinking was demonstrated.
\end{abstract}

Keywords: Social network, collaborative learning, active methodology, participation.

\section{Resumen}

Se utilizó la red social profesional LinkedIn como una plataforma común de exposición, discusión y análisis de contenidos. Su uso puede facilitar el aprendizaje colaborativo de los alumnos, estimular su motivación y potenciar su proyecto de carrera profesional. La experiencia se llevó a cabo 
Uso de LinkedIn como herramienta docente en el Máster de Producción Animal

en tres asignaturas obligatorias del Máster en Producción Animal de la Universitat Politècnica de València durante el curso 2014-2015. Los objetivos específicos fueron: i) crear una comunidad de aprendizaje virtual, ii) facilitar la participación activa de los alumnos, iii) fomentar la interacción profesor-alumno y alumno-alumno, iv) potenciar el pensamiento crítico y v) potenciar el uso de una red profesional que favorezca el proyecto de carrera profesional de los alumnos. Para ello se establecieron 4 acciones basadas en la creación y mantenimiento de un grupo LinkedIn y el desarrollo de una rúbrica de evaluación del pensamiento crítico. La consecución de los objetivos se evaluó mediante la propia actividad de la red, así como evaluaciones cuantitativas y cualitativas y una encuesta de autopercepción. Todos los alumnos crearon y utilizaron su perfil de forma regular. Se propusieron temas de debate, valorando la participación del alumno durante la experiencia. Igualmente, quedó evidenciada la dificultad de evaluar el desarrollo del pensamiento crítico.

Palabras clave: Redes sociales, aprendizaje colaborativo, metodología activa, participación

\section{Introducción}

El uso de redes sociales en educación universitaria es cada vez más habitual (Espuny et al., 2011). Entre estas redes sociales se encuentra la red profesional LinkedIn, orientada a establecer contactos de tipo laboral (networking). Son muy escasas las referencias relativas al uso de esta red social en educación universitaria. Sin embargo, son cada vez más las empresas que seleccionan personal utilizando esta red social. Por tanto, su uso como herramienta educativa puede permitir alcanzar importantes objetivos docentes como fomentar el trabajo colaborativo, el emprendedurismo y el espíritu crítico (Montoneri, 2013), que serán muy útiles para los alumnos de Máster en Producción Animal de la Universitat Politècnica de València (UPV).

Por otra parte, el desarrollo del pensamiento crítico es una competencia transversal esencial que debería desarrollarse en su máximo nivel en los estudios de Máster. Esta competencia ha sido ampliamente estudiada en los distintos niveles educativos (Paul y Elder, 2005). Así, la UPV la considera una de las 13 dimensiones competenciales (DC9), definiéndola como "Desarrollar un pensamiento crítico interesándose por los fundamentos en los que se asientan las ideas, acciones y juicios, tanto propios como ajenos”. El desarrollo de estas competencias transversales forma parte de un ambicioso programa institucional de la UPV que pretende incorporarlas a distintos niveles en grados y másteres. Este programa se corresponde con el primero de los objetivos estratégicos de la UPV 2015-2020. Por tanto,

(c)) EY-NC-ND 2015, Universitat Politècnica de València 
María Cambra-López, Fernando Estellés Barber, Juan José Pascual Amorós, Cristofol Peris Ribera, Salvador Calvet Sanz

este estudio se sitúa en un contexto claramente propicio al uso de nuevas tecnologías como recurso para desarrollar estas competencias transversales.

\section{Objetivos}

Este estudio persigue los siguientes cinco objetivos:

1. Crear una comunidad de aprendizaje virtual en LinkedIn

2. Facilitar la participación activa de los alumnos en la exposición, discusión y análisis de contenidos

3. Fomentar la interacción profesor-alumno y la interacción alumno-alumno

4. Potenciar el pensamiento crítico y multiplicar la diversidad de los conocimientos y las experiencias que adquieran los alumnos como consecuencia del uso dirigido de los debates en el grupo LinkedIn

5. Potenciar el uso de una red profesional que favorezca el proyecto de carrera profesional de los alumnos.

\section{Desarrollo de la innovación}

Para conseguir los objetivos se planificaron las siguientes tareas:

1. Creación de un grupo LinkedIn del proyecto

Durante la primera semana del curso académico 2014-2015 se creó un grupo de LinkedIn que fue administrado por los profesores, con acceso a los alumnos del Máster de Producción Animal (14 alumnos). Éstos crearon su perfil en esta red social (en caso de no tenerlo) y se dieron de alta en el grupo durante la primera semana del cuatrimestre.

2. Mantenimiento del grupo LinkedIn durante el curso

Durante dos meses se llevó a cabo un mantenimiento semanal del perfil LinkedIn por parte de los profesores de tres asignaturas de la titulación, creando una actividad basal centrada en la publicación de contenidos y creación de debates relacionados con las mismas. Las tres asignaturas implicadas fueron: Contaminación Ambiental y Bioseguridad, Ingeniería Medioambiental en Explotaciones Ganaderas y Análisis de Datos en Producción Animal. Se solicitó a los alumnos participar en los debates creados por el profesor, lo cual fue valorado de forma cuantitativa (número de aportaciones) y cualitativa (contribución al debate). Además, se animó a los alumnos a establecer nuevos debates, siempre que estuvieran relacionados con las temáticas tratadas en las asignaturas.

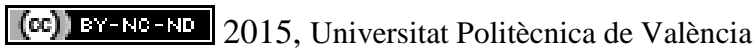

Congreso In-Red (2015) 
3. Desarrollo y aplicación de una rúbrica de evaluación del pensamiento crítico

Con el fin de alcanzar una evaluación objetiva de la adquisición del pensamiento crítico a través del uso de LinkedIn, se realizó una búsqueda bibliográfica para desarrollar una rúbrica objetiva adaptada a cada actividad relacionada con esta competencia transversal.

4. Coordinación y seguimiento del proyecto

El coordinador del grupo LinkedIn revisó semanalmente las aportaciones de los alumnos con el fin de moderar los debates en curso si fuera necesario, aportar nueva información o animar a la participación. Igualmente, se presentarán los resultados de este proyecto en la Comisión Académica del Máster, con el objetivo de establecer estrategias de continuidad en próximos cursos académicos.

\section{Resultados}

Los resultados obtenidos en funión de los objetivos propuestos se presentan a continuación:

Objetivo 1. Crear una comunidad de aprendizaje virtual en LinkedIn

Tal como se recogió en una encuesta inicial, sólo tres alumnos manifestaron tener un nivel intermedio en el manejo de LinkedIn. Los 14 alumnos matriculados se crearon un perfil en esta red social profesional y se dieron de alta en el grupo en un plazo de una semana.

Objetivos 2 y 3. Facilitar la participación activa de los alumnos y fomentar la interacción

Las participaciones de los alumnos en el grupo LinkedIn se resume en ocho publicaciones y 55 comentarios en dos meses. Todos los alumnos participaron, valorándose la calidad de las aportaciones de forma cualitativa: "Excelente" (aporta nueva información elaborada y comentada, contribuyendo al crecimiento de la discusión e incentivando interacciones de otros compañeros); "Bien" (manifiesta su opinión, debidamente fundamentada, pero sin contribuir al crecimiento de la discusión); "Mal" (comentarios sin relación con el tema, o incorrectos en cuanto a su contenido); o "No evaluable" (comentarios que no aportan contenido evaluable a la discusión, sin ser incorrectos o fuera de tema). Si bien el grupo arrancó bien, se evidenció un cierto cansancio por parte de los alumnos con el paso del tiempo, especialmente durante las últimas semanas del proyecto.

Objetivo 4. Potenciar el pensamiento crítico

Se ha buscado información sobre rúbricas para evaluar el pensamiento crítico. Esta búsqueda se ha archivado en un repositorio del grupo de trabajo. No se ha desarrollado aún una rúbrica definitiva dado que los profesores han evidenciado una gran complejidad en la naturaleza (y por tanto en la evaluación) de esta competencia. Si bien inicialmente se pretendía desarrollar una rúbrica para cuantificar en qué medida el uso de LinkedIn 
María Cambra-López, Fernando Estellés Barber, Juan José Pascual Amorós, Cristofol Peris Ribera, Salvador Calvet Sanz

favorecía el desarrollo del pensamiento crítico, durante el estudio se evidenció el carácter multifactorial de esta competencia, y la dificultad de evaluar objetivamente su desarrollo como consecuencia de las actividades realizadas.

Objetivo 5. Impacto sobre la carrera profesional

Actualmente todos los alumnos tienen un perfil de Linkedin activo. A final del cuatrimestre se pasará un cuestionario sobre la proyección profesional que pueda haber tenido hasta el momento.

\section{Conclusiones}

Del presente estudio se extraen las siguientes conclusiones:

1.- La red profesional LinkedIn sirve como foro de discusión y evaluación docente, a pesar de no estar diseñada para tal fin.

2.- Esta red puede permitir la interacción asíncrona entre los alumnos y con los profesores, de forma similar a otras redes, favoreciendo el aprendizaje colaborativo. Permite desarrollar debates paralelos a las clases presenciales, favoreciendo ciertas actitudes por parte de los alumnos que son más difíciles de expresar en una clase presencial.

3.- La evaluación del pensamiento crítico supone una extrema complejidad por la propia naturaleza de esta competencia genérica. Los autores no han sido capaces de demostrar si el desarrollo de esta actividad ha contribuido al desarrollo de esta competencia.

4.- La evaluación del impacto de esta red social en la carrera profesional de los egresados debe plantearse al menos a medio plazo.

\section{Referencias}

AACU (2015). Critical Thinking Value Rubric. Association of American Colleges and Universities. Accesible on-line https://www.aacu.org/value/rubrics/critical-thinking (abril de 2015).

COOPER, B., \& NAATUS, M. K. (2014). Linkedin As A Learning Tool In Business Education. American Journal of Business Education (AJBE), 7(4), 299-306.

ESPUNY, C., GONZÁLEZ, J., LLEIXÀ, M., GISBERT, M. (2011). Actitudes y expectativas del uso educativo de las redes sociales en los alumnos universitarios. Monográfico «El impacto de las redes sociales en la enseñanza y el aprendizaje». Revista de Unidad y Sociedad del Conocimiento, RUSC $8 \mathrm{n}^{\circ} 1$.

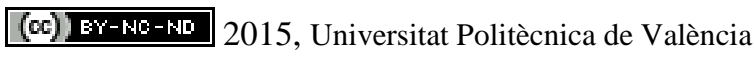

Congreso In-Red (2015) 
LOFGREN, E. A. (2014). Social media and equine science: The effect of LinkedIn on class engagement of equine higher education students (Doctoral dissertation, MURRAY STATE UNIVERSITY).

MONTONERI, B. (2013). Impact of LinkedIn on Boosting Students' Learning Motivation and Career Prospects. COLLA 2013 : The Third International Conference on Advanced Collaborative Networks, Systems and Applications.

MORAN, M. SEAMAN, J., TINTI-KANE, H. (2011). Teaching, learning and sharing: how today's higher education faculty use media. Pearson Learning solutions.

PAUL, R., ELDER, L. (2005). Estándares de competencia para el pensamiento crítico. Estándares, Principios, Desempeño, Indicadores y Resultados. Con una Rúbrica Maestra en el Pensamiento Crítico. Fundación para el pensamiento crítico, 66 pag. 